\title{
PLUME CHARACTERIZATION OF A LABORATORY MODEL 22 N GPIM THRUSTER VIA HIGH-FREQUENCY RAMAN SPECTROSCOPY
}

\author{
George J. Williams ${ }^{1}$ and Jun J. Kojima ${ }^{2}$ \\ Ohio Aerospace Institute, NASA GRC, Cleveland, OH 44135 \\ Lynn A. Arrington, ${ }^{3}$ Matthew C. Deans, ${ }^{4}$ and Brian D. Reed ${ }^{4}$ \\ NASA Glenn Research Center, Cleveland, OH 44135 \\ McKenzie I. Kinzbach ${ }^{5}$ \\ University of Cincinnati, Cincinnati, OH 45221 \\ Christopher H. McLean ${ }^{6}$ \\ Ball Aerospace and Technologies Corporation, Boulder, CO 80301
}

The Green Propellant Infusion Mission (GPIM) will demonstrate the capability of a green propulsion system, specifically, one using the monopropellant, AF-M315E. One of the risks identified for GPIM is potential contamination of sensitive areas of the spacecraft from the effluents in the plumes of AF-M315E thrusters. Plume characterization of a laboratory-model $22 \mathrm{~N}$ thruster via optical diagnostics was conducted at NASA GRC in a space-simulated environment. A high-frequency pulsed laser was coupled with an electron-multiplied ICCD camera to perform Raman spectroscopy in the near-field, low-pressure plume. The Raman data yielded plume constituents and temperatures over a range of thruster chamber pressures and as a function of thruster (catalyst) operating time. Schlieren images of the near-field plume enabled calculation of plume velocities and revealed general plume structure of the otherwise invisible plume. The measured velocities are compared to those predicted by a two-dimensional, kinetic model. Trends in data and numerical results are presented from catalyst mid-life to end-of-life. The results of this investigation were coupled with the Raman and Schlieren data to provide an anchor for plume impingement analysis presented in a companion paper. The results of both analyses will be used to improve understanding of the nature of AF-M315E plumes and their impacts to GPIM and other future missions.

\section{Introduction}

$\mathrm{T}$ HE Green Propellant Infusion Mission (GPIM) is a Technology Demonstration Mission (TDM) project, sponsored by NASA's Space Technology Mission Directorate (STMD). ${ }^{1}$ The goal of GPIM is to demonstrate the capability of a "green" propulsion system, specifically, one using the monopropellant AF-M315E. Green monopropellants offer lower toxicity and reduced safety hazards, while also aiming to provide higher performance than the current state of the art fuels. The GPIM propulsion system will be flown as a payload on a Ball Aerospace BCP-100, a small, standardized spacecraft. The propulsion system was to have one $22 \mathrm{~N}$ thruster for primary divert maneuvers and four $1 \mathrm{~N}$ thrusters for attitude control.

\footnotetext{
1 Senior Scientist, OAI, Propulsion and Propellants Branch, 21000 Brookpark Rd., MS 16-1, AIAA Associate Fellow.

2 Principal Scientist, OAI, 21000 Brookpark Rd., MS-OAI, AIAA Senior Member.

${ }^{3}$ Aerospace Engineer, Aerospace Experiment, Facilities and Test Branch, 21000 Brookpark Rd., MS 35-1, AIAA Member.

${ }^{4}$ Aerospace Engineer, Surface Propulsion Branch, 21000 Brookpark Rd., MS 301-3, AIAA Member.

5 Undergraduate Student, Dept. of Aerospace Engineering, AIAA Student Member.

${ }^{6}$ GPIM Principal Investigator, Mission Systems Engineering, 1600 Commerce Street, AIAA Senior Member.
} 
One of the risks identified for GPIM is potential contamination of sensitive areas of the spacecraft from the effluents in the plumes of AF-M315E thrusters. The plume risk mitigation activities include modeling the plume flow fields of the AF-M315E thrusters, assessing the plume impingement on the BCP-100 spacecraft including the impact on the power generating capabilities of the solar arrays, and conducting ground-based plume measurements on an AF-M315E thruster to correlate the plume modeling with plume data.

The Raman diagnostic was designed to interrogate the plume of a high-expansion area nozzle with exit plane pressures on the order of $0.1 \mathrm{psia}$. This environment has proven very difficult to achieve Raman signals in the past because the signal strengths are proportional to the density. ${ }^{2,3}$ Most Raman combustion diagnostics are implemented at or above atmospheric pressure suggesting a two or three order of magnitude decrease in signal using conventional approaches. In addition to significantly lowering the pressure, nozzle expansion also reduces the temperature of the target gas well below where Raman diagnostics typically interrogate combustion. This removes many of the ro-vibrational characteristics that are used to quantify both temperature and species concentrations. In previous experiments, high-power pulsed lasers were required to achieve modest signal to noise ratios and focusing these lasers to a very small interrogation volume posed a significant hazard to the windows of the vacuum chamber. In this investigation, a high-frequency laser was coupled with an electron-multiplied ICCD (EMCCD) camera to mitigate the constraints imposed by highpower, single-shot lasers and allows accumulation of multiple shots on the ICCD in preference to ensemble averaging.

The objectives of this investigation include identifying the major constituents in the AF-M315E plume and correlating them to variations in chamber pressure, time on catalyst, and spatial position in the plume. These data will be included in a plume-impingement analysis. ${ }^{4}$ Also required for that analysis are velocities in the plume which will obtained via schlieren imaging. The images may also reveal any significant radial variation in plume structure associated with non-uniform combustion. This investigation also serves as the demonstration of capability for the diagnostics needed to interrogate plumes from higher, more flight-like, expansion ratio nozzles with AF-M315E or other green propellants.

\section{Theoretical Background}

The following overview of Raman scattering theory, Schlieren diagnostic technique and VIPER modeling is intended to facilitate understanding of the data and calculations discussed in the next section. More thorough presentations are available in the literature and are noted where applicable.

\section{A. Raman Diagnostic}

The theory of Raman scattering in diatomic and polyatomic molecules is well established. ${ }^{5}$ According to Placzek's polarization theory of the Raman effect, the corresponding differential Raman scattering crosssection per molecule at thermal equilibrium is given by ${ }^{5}$

$$
\left(\frac{\partial \sigma}{\partial \Omega}\right)_{\mathrm{i} \rightarrow \mathrm{f}}^{\theta}=\left(\frac{\pi}{\epsilon_{0}}\right)^{2}\left(v_{0} \pm v_{\mathrm{k}}\right)^{4} \mathrm{~g}_{\mathrm{i}} \frac{\exp \{-\mathrm{E} / \mathrm{kT}\}}{\mathrm{Q}(\mathrm{T})} \Phi\left(\alpha^{2}, \gamma^{2}, \theta\right)
$$

Here, the superscript $\theta$ indicates the relative angle of the planes of polarization between scattered and incident light; the subscripts of $i$ and $\mathrm{f}$ stand for the initial and final states respectively; $\varepsilon_{\mathrm{o}}$, is the vacuum permittivity; $v_{o}$ is incident light (laser) frequency; + and - signs refer to anti-Stokes and Stokes Raman scattering respectively; $n_{k}$ is the molecular vibrational frequency associated with the rotation-vibration transition from $i$ to f, $g_{i}$ is the total degeneracy; $E_{i}$ is the energy of the initial state; $k$ is the Boltzmann constant; $\mathrm{T}$ is the gas temperature; $Q(T)$ is the total partition function of the molecule at temperature $T ; \Phi(\alpha, \gamma, \theta)$ is a function of the mean value of the derived polarizability tensor $\alpha^{2}$, the anisotropy $\gamma$, and $\theta$ is the observation angle that stands for the molecule dependent temperature-independent invariant, which is tabulated by Long ${ }^{5}$ for every branch of ro-vibration and pure rotation scatterings. Note that the factors $(v+1) b_{n}{ }^{2}$, and $v b_{n}{ }^{2}$ should be multiplied to Eq. (1) for ro-vibration Stokes and anti-Stokes scattering of diatomic molecule respectively, here $v$ is the vibrational quantum number and $b_{n}{ }^{2}$ is defined as $\mathrm{h} / 8 \pi^{2} \mathrm{cn}_{\mathrm{k}}$ where $\mathrm{h}$ is the Plank constant and c is the speed of light. The Raman spectral line locations, given by molecule vibrational frequency $v_{\mathrm{k}}$, are calculated on the basis of energy conservation as 


$$
v_{\mathrm{k}}=\frac{\mathrm{E}_{\mathrm{f}}-\mathrm{E}_{\mathrm{i}}}{\mathrm{hc}}
$$

where $E_{\mathrm{f}}$ is the energy of the final state. The energy in a vibrational-rotational state $(n, \mathrm{~J})$ is expressed as the sum of the vibrational energy term, $G(v)$ and rotational energy term, $F(n, \mathrm{~J})$

$$
\mathrm{E}(v, \mathrm{~J})=\frac{\mathrm{G}(v)+\mathrm{F}(v, \mathrm{~J})}{\mathrm{hc}}
$$

According to the anharmonic oscillator model, $G(v)$ of diatomic molecules may be given by ${ }^{6}$

$$
\mathrm{G}(v)=\omega_{\mathrm{e}}\left(v+\frac{1}{2}\right)-\omega_{\mathrm{e}} \chi_{\mathrm{e}}\left(v+\frac{1}{2}\right)^{2}+\omega_{\mathrm{e}} \gamma_{\mathrm{e}}\left(v+\frac{1}{2}\right)^{3}
$$

Here $\omega_{e}, \omega_{e} \chi_{e}$, and $\omega_{e} \gamma_{e}$ are molecular constants and tabulated by Hertzberg. ${ }^{78}$ According to the non-rigid rotator model that takes the influence of centrifugal force due to vibration into account, the rotational term for the singlet ${ }^{1} \Sigma$ state of diatomic molecules such as $\mathrm{N}_{2}$ or $\mathrm{H}_{2}$ may be given by

$$
F(v, J)=B_{v} J(J+1)-D_{v} J^{2}(J+1)^{2}
$$

where $B_{\mathrm{n}}=B_{\mathrm{e}}-\alpha_{e}(n+1 / 2)$, and $\mathrm{D}_{\mathrm{n}}=D_{\mathrm{e}}+\beta_{\mathrm{e}}(n+1 / 2)$ where $B_{\mathrm{e}}, D_{\mathrm{e}}, \alpha$, and $\beta_{\mathrm{e}}$ are molecular constants, which are tabulated by Hertzberg. ${ }^{7}$ Thus for a Stokes Q branch, that is $A n=n_{\mathrm{f}^{-}} n_{\mathrm{i}}=+1$ and AJ $=J_{f^{-}} J_{i}=0$, Raman line frequency is given by

$$
v_{k}(v, J)=\frac{E\left(v_{i}+1\right)-E\left(v_{i}\right)}{h c}
$$

It should be noted that Q-branch of ro-vibrational Raman line is much stronger than the 0- and S-branch because the polarizability tensor greatly exceeds the anisotropy invariant. So we calculate only Q-branch of ro-vibrational Raman scattering for diatomic molecule in the present model.

For S-branch (Stokes) of $\mathrm{H}_{2}$ rotational Raman, the function $\Phi\left(\alpha^{2}, \gamma^{2}, \theta\right)$ is proportional to the anisotropy, $\gamma^{2}$ and the following Placzek-Teller coefficients ${ }^{5}$

$$
\mathrm{b}_{\mathrm{J}+2, \mathrm{~J}}=\frac{3(\mathrm{~J}+1)(\mathrm{J}+2)}{2(2 \mathrm{~J}+1)(2 \mathrm{~J}+3)}
$$

Also, $E(J)=F(J) / h c$ in Eq. (3) where $F(J)=B_{e} J(J+1)-D_{e} J^{2}(J+1)^{2}, v_{k}=E\left(J_{i}+2\right)-E\left(J_{i}\right)$ in Eq. $(6)$, and $\mathrm{Q}(\mathrm{r})=\mathrm{Q}_{\text {rot }}$ in Eq.(7) should be replaced.

Thus, Raman line frequencies and cross-sections of ro-vibrational $\mathrm{H}_{2}$ and pure rotational $\mathrm{H}_{2}$ were calculated using above expressions. Note that we calculated the relative (but quantitative) intensity instead of absolute number by setting the function $\Phi$ and molecular independent constants as a constant number so that the calculated Raman intensity can be fitted to experimental data of each molecule individually to determine temperature.

The intensity I of the Raman lines follows from ${ }^{9}$

$$
\mathrm{I}=\mathrm{k} \Omega \frac{\partial \sigma}{\partial \Omega} \mathrm{nlI}_{0}
$$

Here $n$ is the molecular number density of the respective gas species in the measurement volume, $\Omega$ is the solid angle observed, $l$ is the length of the measurement volume, and $\mathrm{I}_{\mathrm{o}}$ is the laser intensity. Constant $k$ is a setup constant and takes into account the geometry and wavelength-dependent losses. The differential Raman cross section $\frac{\partial \sigma}{\partial \Omega}$ depends on molecule-specific parameters such as the isotropy and anisotropy of the derived polarizability tensor and also has a dependence on the geometry of the setup. For the configuration used here, a polarized excitation laser with polarization vertical to the scattering plane and observation of the scattered signal perpendicular to the laser propagation direction, the scattering cross section of the signal with the vertical polarization direction can be deduced by assumption of a Boltzmann distribution to be

$$
\left(\frac{\partial \sigma}{\partial \Omega}\right)_{\perp}=\operatorname{Cg}_{\mathrm{i}} \frac{\left(v_{0}-\Delta v_{\mathrm{R}}\right)^{4}}{\Delta v_{\mathrm{R}}\left[1-\exp \left\{-\mathrm{hc} \Delta v_{\mathrm{R}} / \mathrm{kT}\right\}\right]}\left[\left(\mathrm{a}_{\mathrm{t}}^{\prime}\right)^{2}+\frac{4}{45}\left(\gamma_{\mathrm{t}}^{\prime}\right)^{2}\right]
$$


For the horizontal polarization component one obtains

$$
\left(\frac{\partial \sigma}{\partial \Omega}\right)_{\|}=\operatorname{Cg}_{\mathrm{i}} \frac{\left(v_{0}-\Delta v_{\mathrm{R}}\right)^{4}}{\Delta v_{\mathrm{R}}\left[1-\exp \left\{-\mathrm{hc} \Delta v_{\mathrm{R}} / \mathrm{kT}\right\}\right]}\left[\frac{1}{15}\left(\gamma_{\mathrm{t}}^{\prime}\right)^{2}\right]
$$

where $\mathrm{C}$ is a collection of several molecule-independent physical constants. For each vibration $i, g_{\mathrm{i}}$ is the degeneracy; $a_{i}^{\prime}$ and $\gamma_{\mathrm{i}}^{\prime}$ denote the mean values of the isotropy and the anisotropy, respectively, of the derived polarizability tensor. For linear molecules and in particular for two-atom molecules in general it is essential that $45\left(a_{\mathrm{i}}^{\prime}\right)^{2} \gg 4\left(\gamma_{i}^{\prime}\right)^{2}$. For this reason the vertical polarization direction of the Raman signals of the vibration $Q$ branch is more intense; i.e., the Raman effect essentially retains the polarization of the incoming laser beam. In contrast, fluorescence emission does not retain the laser polarization because of the relatively long time during which the molecule exists in the excited state before fluorescing. During this time the molecules rotate, eliminating any correlation between the polarization of absorbed and emitted photons. Thus, fluorescence signal contributions can be suppressed by use of a polarization sensitive detection scheme. ${ }^{9}$

A spectral profile of the Raman spectrum at atmospheric pressure is typically modeled using Gaussian profiles, neglecting collisional-broadening effects. ${ }^{10}$ This assumption is even more valid at the low pressures of this investigation. However, both the laser and the spectrometer contribute to (broaden) the observed line shape of the Raman signal. The natural broadening laser and spectrometer convolve to form an overall Gaussian width of ${ }^{11}$

$$
\Delta v_{G}(v, J)=\left(\Delta v_{D}^{2}+\Delta v_{\text {Laser }}^{2}+\Delta v_{\text {Spec }}^{2}\right)^{0.5}
$$

Where, $\Delta v_{D}$ is the molecular-dependent Doppler width, $\Delta v_{\text {Laser }}$ is the laser line width, $0.003 \mathrm{~cm}^{-1}$, and $\Delta v_{\text {Spec }}$ is the spectral resolution of the spectrograph, $14.0 \mathrm{~cm}^{-1}$. For temperatures between $500{ }^{\circ} \mathrm{C}$ and 1000 ${ }^{\circ} \mathrm{C}, \Delta v_{\text {Spec }} \gg \Delta v_{D} \gg \Delta v_{\text {Laser }}$. The profile of Raman spectra at the temperature, $\mathrm{T}$, was calculated by integration (sum) of all lines based on the assumption of the additive approximation.

In order to quantitatively analyze the contribution of individual species in the overall Raman spectra, the relative scattering intensities of each of the different species had to be realized and properly implemented in the calculations. An empirical scaling factor for the scattering intensity of a rotational or vibrational Raman spectrum of species $\mathrm{j}$ relative to the $\mathrm{H}_{2} \mathrm{O}$ Raman scattering intensity can be defined as ${ }^{12}$ :

$$
\Gamma_{j}=\frac{\sum_{a=a}^{b} I_{j} / N_{j}(p, T)}{\sum_{a=c}^{d} I_{H_{2} \mathrm{O}} / N_{\mathrm{H}_{2} \mathrm{O}}(p, T)}
$$

Where $I_{j}$ is the pixel intensity (CCD count per pixel) of a measured rotational or vibrational Raman spectrum of species $\mathrm{j}, \mathrm{n}$ is the pixel number and $\mathrm{a}, \mathrm{b}, \mathrm{c}$, or $\mathrm{d}$ indicate a corresponding pixel range for the summation of the spectrum of species $\mathrm{j}$, and $\mathrm{N}_{\mathrm{j}}(\mathrm{p}, \mathrm{T})$ is the calculated chemical-equilibrium number density of species $\mathrm{j}$ for a given pressure and temperature. $\mathrm{H}_{2} \mathrm{O}$ was chosen as the normalizing species because its signal is free from the interference of the other major species in the plume.

The data were reduced using a deconvolution routine written in Mathematica. While incorporation of $\Delta v_{\text {Spec }}$ was straightforward to shape the spectra for each rotational and vibrational transition, the masking of temperature dependence (captured in $\Delta v_{D}$ ) precluded use of this model to estimate the temperature via line shape. Because of the relatively large spacing between the ro-vibrational $\mathrm{H}_{2}$ Raman spectra (i.e. $\gg \Delta v_{\text {Spec }}$ ), it was possible to estimate the (vibrational) temperature by fitting this spectra with peaks calculated via Eqns. 3, 5-7. The spectra lay between $665 \mathrm{~nm}$ and $690 \mathrm{~nm}$, which is also largely free of overlap with Raman spectra from other major species. Within the error of measurement, the vibrational temperature will correspond to the kinetic/equilibrium temperature of the plume gas mixture.

\section{B. Sschlieren Diagnostcs}

Optical inhomogeneities refract light rays in proportion to their gradients of refractive index, $\mathrm{n}$, in an $\mathrm{x}, \mathrm{y}$-plane. ${ }^{13}$ Strong optical disturbances (such as shocks) will mask changes behind them. Depending on the degree of pressure matching, this may be true for Prandtl-Meyer expansion fans as well. However, the 
boundary transition of the plume should yield data to approximate the velocity field if not the density field. The boundary of the plume can be characterized by its radius with respect to centerline and its local tangent angle, $\Phi=\delta+v_{e}-v_{n}$, where $\delta$ is the nozzle exit angle and $v_{\mathrm{e}}$ and $v_{\mathrm{n}}$ are the Prandtl-Meyer angles at the nozzle exit and after expansion, respectively. ${ }^{14} \delta$ and $v_{\mathrm{n}}$ are constant for a given nozzle and $\Phi$ is taken from a Schlieren image. The local Mach number can then be determined from ${ }^{15}$

$$
v_{e}=\sqrt{\frac{\gamma+1}{\gamma-1}} \tan ^{-1} \sqrt{\frac{\gamma+1}{\gamma-1}\left(M^{2}-1\right)}-\tan ^{-1} \sqrt{M^{2}-1}
$$

Unfortunately, the ratio of specific heats $\gamma$, and the stagnation value of the density are unknown. They are approximated using the numerical modeling described below.

The schlieren images were reduced using Matlab. Color images were first converted to greyscale for mathematical manipulation. A background (pre-firing) image was subtracted from each image taken during the thruster's operation. Several images were then averaged and the contrast of the average was maximized. Plume angles, $\Phi$, were determined by manual curve fitting of the final images.

\section{TDK-VIPER Modeling}

The Viscous Interaction Performance Evaluation Routine (VIPER) Version 3.6.2 (updated 2008) was used to model the expansion of the combustion gases from the exit of the catalyst bed to a slight distance downstream of the nozzle exit plane. The VIPER code is a modified version of the Two-Dimensional Kinetics (TDK) code with a Parabolized Navier Stokes (PNS) solver. ${ }^{16}$ The modeling effort was undertaken to correlate the optically measured plume properties to pre-expansion (combustion chamber equivalent) values which could be used as inputs in the parallel effort to model the plume impingement. The model also provided a qualitative assessment tool for the concentration measurements.

Initial temperature and pressure were taken to be experimentally measured values. The initial concentrations were adjusted to yield the observed concentrations in the near-field plume. No radial zoning was included as no variation in radial conditions could be tied to catalyst bed flow without post-test inspection of the catalyst bed and bed plate. Inert and slowly-reacting constituents were incorporated to simulate partial or off-nominal combustion. No liquid species were assumed.

In principle, VIPER has the capability to extend the plume calculations downstream of the nozzle. However, specifying the appropriate mesh and viscous parameters proved impractical. Instead, a virtual nozzle corresponding to the plume boundary shown in the Schlieren images was used. This allowed calculation of the plume properties several nozzle diameters downstream. The impact of this approximation on centerline should be negligible, however, predictions near the edges of the plume will not include the transitional, viscous region of the real plume.

\section{Experimental Configuration}

This section describes the apparatus used to collect the Raman and Schlieren data. Brief descriptions are also provided for the thruster and test facility.

\section{A. GPIM Laboratory-model Thruster}

A lab model $22 \mathrm{~N}$ was provided by Aerojet-Rocketdyne for testing at NASA-GRC. The lab-model thruster incorporated a conical 8:1 nozzle with an exit pressure on the order of a few psia. This underexpanded condition in the RCL-chamber provided a target environment with identical plume constituents at $10 \mathrm{x}$ the pressure of the more flight-like expansion of a higher-fidelity thruster which has a pressure-matched nozzle. A more detailed description of the thruster is provided in an accompanying paper. ${ }^{17}$

Following a test series at Aerojet-Rocketdyne, Redmond, the thruster arrived at GRC with roughly half of its projected catalyst life remaining. Laser diagnostics did not begin until the thruster had completed additional acceptance testing at GRC, and, therefore, laser-based data collection began well into the mid-life of the thruster's catalyst. 
The thruster was typically operated in one of two modes during this test campaign. A pulse train of ten $0.5 \mathrm{~s}$ pulses began and ended each test day as a thruster health check. The vast majority of the remainder of the segments were continuous $4 \mathrm{~s}$ firings with a few longer and a few shorter included to investigate specific trends. Chamber pressure and temperature measured downstream of the catalyst bed were recorded for each operating point as function of time at a rate of $4 \mathrm{~Hz}$. This allowed spectroscopic and operational data to be correlated at several points during a firing segment.

\section{B. Altitude Simulation}

Plume measurements of an AF-M315E thruster were conducted at GRC's Research Combustion Laboratory - Cell 11 (RCL-11). RCL-11 is a small rocket $(<220-\mathrm{N})$, altitude $(36.6 \mathrm{~km})$ facility designed with optical access to incorporate the use of laser-based diagnostics. As shown in Fig. 1, the facility uses a six- foot long, three-foot diameter cylindrical vacuum tank. Thrust was measured via load cells. Vacuum is achieved by the use of a two-stage ejector system driven by motive air supplied from a central GRC facility. The thruster is fired horizontally into a water-cooled diffuser which provides an additional pumping effect for the vacuum tank. The water cooling system has a two stage dewatering subsystem to maintain the altitude condition of the vacuum tank throughout operation. RCL-11 has been used to test thrusters for up to 1 hour in duration, with the test duration limited by the thruster rather than the vacuum capability.

The test environment in RCL-11 operated with a base pressure around $1720 \mathrm{~Pa}(0.25 \mathrm{psia})$ at quiescent conditions, and was seen to drop to $690 \mathrm{~Pa}(0.10 \mathrm{psia})$ during thruster firing. The chamber backpressure was primarily nitrogen gas, which was being used as a purge gas to cool the thruster injector between firings. The thruster was fired into a water-cooled diffuser with an inlet inner diameter of $8.26 \mathrm{~cm}(3.25 \mathrm{in})$ that was located approximately $6.35 \mathrm{~cm}$ (2.5 in) downstream of the thruster exit plane. Further details of the test cell are described in Deans. ${ }^{18}$

\section{Raman Diagnostic}

As stated above, to circumvent issues associated with high-power laser delivery through vacuum chamber windows a high-frequency laser was coupled with an electron-multiplied ICCD (EMCCD) camera to mitigate the constraints imposed by high-power, single-shot lasers. A $10 \mathrm{kHz}$, frequency-doubled Nd:YAG solid state laser with $88 \mathrm{~ns}$ pulse widths yielded a time-averaged power of $200 \mathrm{~W}$. The relatively low power-per-pulse enabled tight focusing of the beam through the vacuum chamber windows without risk of damage to the windows. The beam was delivered through a series of mirrors and collimating lenses from an adjacent laser room. Once at the test cell, the laser was directed downstream of the nozzle by a mirror and lens mounted on a translation stage which allowed remote positioning of the laser downstream of the nozzle. Figure 2 shows a schematic of the collection optics with respect to the test cell.

Collection of the Raman signal was through a 6-fiber fiber optic cable. An imaging lens enabled each fiber in the cable to collect light from a separate collection volume focused $60 \mathrm{~cm}$ from the lens. The focal volumes were spaced $6.25 \mathrm{~mm}$ apart to simultaneously capture variations across the diameter of a pressurematched nozzle. The under-expanded nozzle used in this investigation yielded a plume of smaller diameter and up to five of the interrogation points lay outside of the plume depending on axial location. This allowed simultaneous measurement of the plume and the ambient gas, shot-to-shot calibration of the laser power, detector drift, and laser-detector alignment. Measurement of signals outside of the plume also provided a path to eliminate facility effects such as window fluorescence. A three stage positioning system remotely translated the collection optics and allowed optimization of the laser-collection optics alignment at atmospheric pressure. Negligible hysteresis was observed in the stages which allowed rapid and repeatable interrogation of locations downstream of the nozzle exit plane.

The multi-fiber cable delivered the Raman scatter to a back-illuminated EMCCD camera via an imaging spectrograph. The PI-MAX4:512EMB used in this investigation fiberoptically coupled the EMCCD to an image intensifier that enabled six times higher light throughput than lens-coupled configurations. The camera potentially allows $1000 \mathrm{x}$ greater sensitivity than standard ICCD cameras. ${ }^{16}$ In addition the intensifier can be gated to the pulse width of the laser at the laser frequency while the EMCCD accumulates tens to over 10000 laser-shots to form a single image. In preparation for testing on the GPIM thruster, Kojima demonstrated 
this technique's ability to capture time-resolved characteristics of an atmospheric hydrogen flame. ${ }^{19}$ The imaging spectrograph allowed each of the fiber's signals to be independently imaged on the camera and thus simultaneously collected through independent accumulations.

A sharp-edge filter suppressed the resonant (Rayleigh) scatter from the plume and all anti-Stokes Raman spectra. Raman spectra from just above $532 \mathrm{~nm}$ to $700 \mathrm{~nm}$ was collected on the camera. This range was sufficient to include rotational and vibrational spectra. Spectral resolution was sacrificed in order to maximize the throughput (signal) of the collection optics. This introduced significant instrument broadening of the Raman signals on the order of several $\mathrm{nm}$. To the extent possible, this broadening was removed in the deconvolution as described above, but significant uncertainty remains due to the overlap of $\mathrm{NO}_{2}, \mathrm{~N}_{2} \mathrm{O}$, and $\mathrm{CO}_{2}$ vibrational and $\mathrm{H}_{2}$ rotational spectra in the $550 \mathrm{~nm}$ to $585 \mathrm{~nm}$ range.

\section{Schlieren Diagnostic}

A coincident configuration was selected for the Schlieren diagnostic to facilitate rapid, remote transition from the schlieren to the Raman diagnostic. This technique utilizes a single primary mirror instead of two as in the common z-type schlieren optics configuration. A schematic of the coincident Schlieren diagnostic configuration is shown in Figure 3. The coincident configuration offers several advantages including a double pass through the target area which, in principle, doubles the sensitivity of the measurement and reduction in experimental footprint with long-focal length mirrors.

Background-oriented Schlieren has been demonstrated to provide the best quantitative analysis of a similar flow field. ${ }^{13,20}$ However, this method was not incorporated because it would have precluded viewing of the thruster by facility monitoring cameras and because the background may have fluoresced during Raman interrogation.

The large turning mirror introduced distortion, but atmospheric and subsequent vacuum data suggested that this effect could be neglected in the determination of the plume shape. The ribbon-filament tungsten lamp provided a wide-band, variable intensity light source. Optics incorporated in the configuration eliminated the ultraviolet and far-infrared light. A 50-cm diameter, 1-meter focal length spherical mirror was used as the primary mirror. A larger diameter mirror would have been preferable, but it would have interfered with both the Raman diagnostic and optical access in general to the thruster. As shown in the schematic, only the primary mirror was located in the vacuum chamber. A low distortion, F4.5-F22C zoom lens with a relatively large depth of field was used to image a region slightly larger than the primary mirror. Optimization of the coupling lens yielded a magnification of roughly 3:1. Baffles were employed to prevent stray light from the tungsten lamp from entering the camera.

\section{Results and Discussion}

Results of the Raman and Schlieren diagnostics are discussed below followed by a correlation of those measurements with the VIPER modeling. The laser had a long beam waist which allowed for collection of data in the vertical direction without correction for laser intensity. This was verified repeatedly during alignment checks which are described below.

The thruster was operated in a manner that facilitated plume interrogation and not in ones that corresponded to either design operation: pulse trains of multiple, short duration pulses or steady-state operation. Typical firings consisted of 4-s pulses which approached steady conditions in pressure and flow near the 4-s point and thus pointed to concentrations that might be expected in truly steady operation. A pair of 8-s firings were included in the test matrix to verify that the concentrations seen at $4 \mathrm{~s}$ were representative of steady operation. Pulse trains of ten 0.5 -s pulses were undertaken as daily health checks of the thruster. As the test campaign progressed, these provided an opportunity to interrogate the plumes associated with this mode of operation as well. 


\section{A. Typical Raman Spectra}

At the beginning and end of each test sequence, Raman data were collected at atmospheric pressure to calibrate the spectra given changes in laser power, window and/or mirror degradation, and spectrograph drift. These calibration checks also allowed for optimization of the Raman signal collection via alignment of the collection volumes with the laser. Data taken post-test at roughly atmospheric pressure had very weak $\mathrm{O}_{2}$ and $\mathrm{H}_{2} \mathrm{O}$ signals as the rise in pressure was mostly due to the $\mathrm{N}_{2}$ facility purge. The two "high-pressure" conditions facilitated calibration and validation of the deconvolution model. Raman spectra were taken at simulated altitude conditions without thruster operation. Similar, slightly weaker, spectra were obtained outside of the plume during thruster operation. These vacuum, no plume data enabled calibration and ensured alignment of the laser and collection volumes. They also facilitated proper adjustment of camera integration and gain settings.

Figure 4 shows a Raman spectrum taken during thruster firing at catalyst mid-life with $\mathrm{p}_{\mathrm{c}}=1.55 \mathrm{MPa}$ (225 psia) and $\mathrm{T}_{\mathrm{C}}=1480 \mathrm{~K}$. As indicated in the figure, major plume species associated with equilibrium combustion $\mathrm{H}_{2} \mathrm{O}, \mathrm{N}_{2}, \mathrm{H}_{2}$, and $\mathrm{CO}_{2}$ are present. The spectrum shown in Fig. 4 resulted from the accumulation of 1000 individual spectra $(0.1 \mathrm{~s}$ total accumulation time). A fit also is shown which is used to deconvolve the various individual spectra. From Eqns. 9 and 10, if the laser's polarization is shifted $90^{\circ}$, the resulting "Raman" spectrum should not contain vibrational-Raman spectra. Figure 5 shows the spectrum resulting from the subtraction of the polarization shifted signal from that in Fig. 4. This spectrum should contain only vibrational-Raman spectra and therefore is better-suited to identifying the major species in the plume.

Repeated collection of atmospheric Raman signals eliminated uncertainty in the ICCD pixel-towavelength correlation. It also removed uncertainty associated with large, day-to-day variations in laser quality (which were small). Impacts due to shot-to-shot variations in laser power were mitigated by accumulating large numbers (100's) of individual shots for each data point and, when possible, averaging several data points to obtain a single spectra. No significant variation in laser power was observed over the course of a day.

However, uncertainties remain in the amplitude of individual peaks in the spectra, in the species associated with peaks in regions of Raman signal overlap, and in the cross sections associated with many of the vibrational Raman spectra necessary for estimating concentrations. Vibrational $\mathrm{N}_{2}, \mathrm{H}_{2} \mathrm{O}$, and $\mathrm{H}_{2}$ Raman spectra present in regions relatively free of other spectra. In addition, the signal to noise ratio for these transitions is greater than 5 for most cases. Therefore, the uncertainty in estimating peak height associated with these species is less than $10 \%$ for $\mathrm{N}_{2}$ and $\mathrm{H}_{2} \mathrm{O}$ and $20 \%$ for $\mathrm{H}_{2}$. As seen in Table 1, the spectra of $\mathrm{CO}_{2}$, $\mathrm{NO}_{2}, \mathrm{~N}_{2} \mathrm{O}$, and $\mathrm{N}_{2} \mathrm{O}_{2}$ lay in the same region and, in part due to instrument broadening, convolve to yield a series of weak peaks with significant uncertainty in the individual contributions of each of these species overall spectra. While tempting to give preference to $\mathrm{CO}_{2}$ as the only equilibrium combustion product in this group, the presence of hydrocarbons in the spectra suggest it is unlikely to be present in concentrations associated with equilibrium. It is impossible a-priori to say which species is more prevalent and non-unique deconvolutions result which match the spectra with 30-40\% uncertainty in each of these species' concentrations. Because the $\mathrm{N}_{2} \mathrm{O}$ cross sections are so large relative to the others, the relative similarity in overall peak strengths suggest its concentrations are always relatively small, so the impact of a large uncertainty in its concentration does not correspond to a similar uncertainty in the concentrations of other species.

Temperature at the point of interrogation was estimated both by fitting the $\mathrm{N}_{2}$ rotational peak and the $\mathrm{H}_{2}$ ro-vibrational spectra. The $\mathrm{N}_{2}$-based estimate was limited by the instrument broadening and by the presence of the large non-Raman signal at $545 \mathrm{~nm}$. The latter was removed via a Gaussian fit to its spectra. Uncertainty in the temperature measurement increased during the test campaign from roughly $10 \%$ due only to instrument broadening to $30 \%$ at the end of testing due to the significant convolution of the $\mathrm{N}_{2}$ and 545 nm spectra. For those conditions in which signal to noise of the $\mathrm{H}_{2}$ was greater than 3 (most of the data after $3 \mathrm{~s}$ during a thruster firing), fitting of the $\mathrm{H}_{2}$ ro-vibrational yielded temperature estimates comparable to the $\mathrm{N}_{2}$ fits. While this was reassuring, it did not reduce the overall uncertainty in temperature because the sensitivity of the ro-vibrational spectra could not resolve temperature changes below roughly $15 \%$. At the end of testing, when the $\mathrm{N}_{2}$-based temperature measurement had an uncertainty greater than this, the $\mathrm{H}_{2}$ spectrum was reduced in magnitude because of the increase in hydrocarbon capture of the hydrogen. 


\section{B. Trends in Concentrations}

The Raman diagnostic was intended to measure the species concentrations and temperature at a couple of steady-state, representative operating conditions and to capture trends in the specie concentrations with chamber pressure, time on catalyst, and as a function of position in the plume. Each of these is discussed below.

Figure 6 compares the measured concentrations for chamber pressures of 170, 200 and 250 psia taken $3 \mathrm{~mm}$ downstream of the nozzle exit plane at catalyst mid-life. Raman data were accumulated for $0.2 \mathrm{~s}$ and three data sets were acquired and averaged over the last second of the 4-s firings at these conditions. However, except for the increase in $\mathrm{X}_{\mathrm{H} 2 \mathrm{O}}$, all changes in concentrations are within the uncertainty of the measurements and suggest that the pressure has little impact on plume constituents over the ranges tested.

Figure 7 shows the variation in specie concentrations during an 8-s firing during catalyst mid-life with $\mathrm{Pc}=230 \mathrm{psia}$ for the final 5 seconds. As alluded to in the above discussion, there was no discernable Raman spectrum in first $1.5 \mathrm{~s}$ of operation. Raman data were accumulated for $0.2 \mathrm{~s}$ and four data sets were acquired and averaged over each second of the last $7 \mathrm{~s}$ of the 8-s firing. (Data points in Fig. 7 represent the centers of these averages.) There are no significant changes in any trend after $4 \mathrm{~s}$ which validates the 4 -s firing as a minimum firing time to reach steady conditions. Between 1.5 and $3.5 \mathrm{~s}$, there are significant changes in the mole fractions of the constituents.

Figure 9 shows spectra taken simultaneously at 6 radial locations $6.25 \mathrm{~mm}$ apart 4-mm downstream of the exit plane for $\mathrm{Pc}=250 \mathrm{psia}$. Data were accumulated for $1 \mathrm{~s}$ on the ICCD and the spectra falling roughly between 2.75 to $3.75 \mathrm{~s}$ were used to approximate the steady-state concentrations. Long integration times were required because larger gains associated with fewer accumulations resulted in cross-talk between the fiber signals. Rotational and fluorescence data have not been removed since three of the interrogation points lie outside of the plume and are dominated by rotational- $\mathrm{N}_{2}$ spectra. Figure 10 compares the calculated mole fractions for these locations. Variations in the concentrations are within the uncertainty of the calculations. Figure 11 shows a slight variation in temperature with radius. Note the temperature outside of the plume suggests some mixing, but the instrument broadening introduces larger error at lower temperatures.

Figure 12 shows the variation in concentrations as a function of axial position along centerline. There is very little variation as would be expected in the high-speed (frozen) flow. Temperature variation with axial position is given in Fig. 13. Temperatures calculated using rotational- $\mathrm{N}_{2}$ spectra and ro-vibrational- $\mathrm{H}_{2}$ spectra are compared in the figure. As the signal decreases away from the exit plane, the $\mathrm{H}_{2}$-based temperature has very large uncertainty. However, at 1 and $3 \mathrm{~mm}$, the temperatures agree within the uncertainty.

To complete the discussion of concentration trends in the catalyst mid-life, Figure 14 shows the typical variation in concentrations during a pulse train of 100.5 -s pulses. Data were accumulated for $0.1 \mathrm{~s}$ and the data falling roughly between 0.35 and $0.45 \mathrm{~s}$ in a pulse were used to characterize the pulse. Trends seen during the multi-second firings above were also observed in both the pulse train and individual pulses. The first pulse was dominated by a non-Raman signal. The next three pulses have large non-Raman signals, but Raman signals are present and relative concentrations of the major species can be approximated. The final six pulses have clean Raman signals and yield quantitative mole fractions. Note that there is a monotonic trend in the $\mathrm{CO}_{2}$ with pulse number. It corresponds to the trend in $\mathrm{Tc}$ as shown in Fig. 15 and to some extent the trends in Fig. 8. Each pulse had a similar non-Raman start and finish as the longer 4-s firings, though both were smaller for the shorter firings.

Pulse trains at catalyst end-of-life showed similar trends to those at catalyst mid-life with the exception that the fluorescent signals remained throughout the pulses and occasionally a mid-train pulse exhibited behavior similar to the first pulse. Figure 16 shows the trends in concentrations as measured by $0.1 \mathrm{~s}$ accumulations between 0.35 and $0.45 \mathrm{~s}$ of $0.5 \mathrm{~s}$ pulses.

The Raman diagnostic proved successful in measuring both specie concentrations and temperatures in the plume of the 22-N laboratory-model thruster. The Raman diagnostic technique of using a high-frequency, relatively low power laser and accumulating many individual spectra on an EM-ICCD was demonstrated to be capable of characterizing the plume of the GPIM lab-model thruster. High signal-to-noise data were collected of the background gas in the test chamber which was roughly at the pressure expected in the plume 
of the EM thruster. This suggests that the Raman technique has the sensitivity to measure the specie concentrations in the plume even at pressures one-tenth that seen in the present investigation.

\section{Schlieren Imaging}

Schlieren imaging was implemented at the end of the test campaign. For assessment of plume structure, the Schlieren images were integrated for $50 \mathrm{~ms}$ and taken in a continuous stream from pre-firing to postfiring. $50 \mathrm{~ms}$ integration provided a compromise between intensity and time resolution. Longer tended to blur the transition region due to averaging of oscillations in the plume boundary (likely corresponding to oscillations in Pc) and shorter exposures yielded weaker overall signals.

Typical raw and reduced Schlieren images are shown in Figure 17. The image is of $3.5 \mathrm{~s}$ into a 4-s firing with $\mathrm{Pc}=200$ psia. Note that there are clear boundaries associated with the viscous layer extending roughly $5 \mathrm{~mm}$ downstream in the image. A core flow is evident as are regions of large gradient near the exit of the nozzle. (The bow-shock-like structure on the right hand side of the image is likely a false image resulting from contamination on one of the mirrors as the structure is present in images without flow. In subtracting the background image, it presents as a white structure.)

A series of Schlieren images in Fig. 18 shows the development of the plume over a 4-s firing with $\mathrm{Pc}=200$ psia. Note that between 0 and $1.3 \mathrm{~s}$ there is a strong plume boundary suggesting reacted, supersonic flow and also a strong core flow. This supports the hypothesis that the large fluorescent signal at the beginning of firing results from gaseous, incomplete combustion including a large amount of soot-like effluent. As the firing progresses, the plume boundary is seen to weaken which is largely due to the large pressure oscillations occurring at this point in the test campaign. At the end of the firing, the flow resembles the pre-firing flow and there is no structure that would support a large scattering from uncombusted propellant. This supports the hypothesis that the end-of-firing fluorescence results from a diffuse flow back into the test chamber from the diffuser.

Figure 19 shows velocity profiles calculated from Schlieren images for operation at $\mathrm{Pc}=110$ and 200 psia. There are large uncertainties in the measured values resulting from the images being slightly out of focus. The trends and magnitudes are, however, consistent with those calculated using the VIPER code.

\section{Correlation of Data to VIPER Modeling}

Flow characteristics upstream of the nozzle exit plane calculated using the VIPER code to match the concentrations and temperatures measured via the Raman technique in the plume. The chamber pressure and temperature were set using experimentally measured values as well. The relative concentrations of the major species are more consistent with equilibrium flow than frozen flow, their values being reduced by the additional carbon, methane, and nitrogen-oxides present. However, the VIPER-predicted plume temperatures were consistently about $300 \mathrm{~K}$ less than those measured. Plume temperatures calculated using the HAP code and identical chamber conditions, ${ }^{17}$ however, agree with those measured.

Unfortunately, the large uncertainty in mass flow rate through the thrust chamber precluded comparisons of predicted and measured performance. However, trends in the predicted and measured performance were consistent. In particular, inclusion of soot and larger hydrocarbons in the gas constituents resulted in performance degradation similar to that seen between mid-life and end-of-life operation.

No effort was made to capture the large fluorescent signals during the start of each firing. However, soot and small hydrocarbons were incorporated upstream of the throat of the nozzle with some success. This suggests that VIPER can be used to model the performance of AF-M315E thrusters given reliable predictions for the plume species as a function of chamber pressure. This investigation provides an advance in that direction. However, higher-resolution Raman data are required at or before catalyst mid-life to support mission planning. 


\section{Future Work}

The diagnostic effort reported in this publication was intended to be a preliminary assessment of the diagnostic techniques for subsequent implementation on a higher fidelity AF-M315E fueled thruster. While the second round of testing was cancelled, several lessons learned in this first test campaign boded well for a more robust characterization of the EM thruster.

\section{A. Improvement in Raman Characterization}

Reduction in the instrument broadening which led to large uncertainties in both the amplitudes and associated species of Raman signals in the $550-580 \mathrm{~nm}$ region can likely be achieved by replacing the grating in the spectrograph. Because of the large signal-to-noise in the background gas, it is projected that the loss in signal with the finer grating can be tolerated. This improvement will allow discrimination between the nitrogen-oxides (typical of HAN combustion/decomposition) and hydrocarbons (typical of HEHN) both when combined in AF-M315E and when tested separately.

\section{B. Improvement in Schlieren Characterization}

Introduction of a higher-fidelity knife edge resulted in a significant gain in signal to noise ratio of the Schlieren diagnostic. In addition, a simple optimization of the alignment removed distortion evident in Figs. 17 and 18. A larger primary mirror was ordered for use in the $2^{\text {nd }}$ series of tests.

Longer integration times were projected for interrogation away from the end-of-life condition. This too will greatly enhance the signal to noise ratio and will likely enable the technique to be applied to the higher expansion ratio nozzle. However, as the nozzle would likely have been pressure matched, the transition from plume to background would have been much more subtle.

\section{Summary and Conclusion}

The novel approach of accumulating a large number of individual Raman spectra generated by a highfrequency laser was demonstrated. This was enabled by the new capabilities of the electron-multiplied ICCD camera. Raman characterization of the plume of a $22 \mathrm{~N}$ laboratory model thruster showed concentrations of major species consistent with predictions based on equilibrium conditions with the exception of added hydrocarbon species. Strong $\mathrm{N}_{2}$ rotational and vibrational Raman signals were obtained even at pressures below 0.1 psia. Significant improvement in the spectral resolution is believed possible through the simple changing of the discriminating grating in the spectrograph without prohibitive losses in signal strength.

Schlieren imaging provided the first structural characterization of an AF-M315E plume. The images indicated plume velocities consistent with VIPER model predictions. However, the resolution of the images was not sufficient to enable quantitative characterization of the 2-dimentional density gradient in the plume. Post-test replacement of the knife-edge yielded much sharper imaging capability which should enable this quantitative characterization in future tests. Qualitative analysis of the plume structure showed the schlieren technique's ability to capture plume oscillations on the timescale of $50 \mathrm{~ms}$.

The VIPER code was successfully modified to predict the kinetic expansion of the AF-315E monopropellant through the thrust chamber and downstream of the nozzle. The latter was accomplished by matching the expansion to the boundary measured via schlieren. Hydrocarbons and nitrogen oxides were included in the non-equilibrium composition and allowed to react. Very little change in concentrations during the expansion were observed.

The test campaign was successful in generating sufficient data to anchor the plume impingement model presented in a companion paper. Through straight-forward modifications, the techniques appear quite capable of characterizing the plumes of both higher-thrust and higher expansion nozzles of thrusters using green-propellants such as AF-M315E or other HAN and HEHN based propellants. 


\section{ACKNOWLEDGMENTS}

The authors thank the entire RCL team at GRC involved with the test setup and data collection. The programmatic support of Tim Smith at NASA GRC is also gratefully acknowledged.

\section{REFERENCES}

\footnotetext{
${ }^{1}$ McLean, C. H., Hale, M. J., Deininger, W. D., Spores, R. A., Frate, D. T., Johnson, W. L., and Sheehy, J. A., "Green Propellant Infusion Mission Program Overview," 49 $9^{\text {th }}$ AIAA/ASME/SAE/ASEE Joint Propulsion Conference \& Exhibit, San Jose, CA, AIAA-2013-3847, July 2013.

2 deGroot, W.A., "Temperature and Species Measurements in a Low-Thrust Hydrogen/Oxygen Rocket Engine," AIAA Journal of Propulsion and Power, Vol. 14, No. 3, May-June 1998, pp. 301-308.

3 deGroot, W.A. and Tsuei, H. H., "Gaseous Hydrogen/Oxygen Injector Performance Characterization," AIAA 94-0220, 32nd Aerospace Sciences Meeting, Reno, NV, January 1994.

${ }^{4}$ Reed, B., et al., “Green Propellant Infusion Mission Plume Impingement Analysis Approach,” JANNAF Conference Paper 2014-3214, May, 2014.

${ }^{5}$ Long, D. A., Raman Spectroscopy, McGraw-Hill, London, (1977).

${ }^{6}$ Hertzberg, G., Molecular Spectra and Molecular Structure - I. Spectra of Diatomic Molecules: 2nd Ed., Krieger Publishing Company, Florida, (1950).
}

${ }^{7}$ K. P. Huber, and G. Hemberg, Molecular Spectra and Molecular Structure - IV. Constants of Diatomic Molecules, Van Nostrand Reinhold Company, New York, (1979).

${ }^{8}$ Hertzberg, G., Molecular Spectra and Molecular Structure - II. Infrared and Raman Spectra of Polyatomic Molecules, Ed., Krieger Publishing Company, Florida, (1950).

${ }^{9}$ A. Leipertz, "Temperaturbestimmung in Gasen mittels linearer und nichtlinearer Raman-Prozesse," Habilitation thesis (Universitat Bochum, Bochum, Germany, 1984).

${ }^{10}$ Kojima, J. and Nguyen, Q-V., "Spontaneous Raman Scattering Diagnostics for High-pressure Gaseous Flames," $22^{\text {nd }}$ AIAA Aerodynamic Measurement Technology and Ground Testing Conference, AIAA Paper 2002-3041, June, 2002.

${ }^{11}$ Eckbreth, A. C., Laser Diagnostics for Combustion Temperature and Species, Ababcus Press, Energy and Engineering Science Series, Gupta, A. K. and Lilley, D. G., ed., Cambrige, MA 1988, pp160-186.

12 Jun, K. and Nguyen, Q-V., "Quantitative Analysis of Spectral Interference of Spontaneous Raman Scattering in High-Pressure Fuel-Rich $\mathrm{H}_{2}$-Air Combustion," Journal of Quantitative Spectroscopy 5 (4) 2004 pp 3033-3050.

${ }^{13}$ Settles, G. S., Schlieren and Shadowgraph Techniques, Springer, New York, 2001.

${ }^{14}$ Elsinga, G. E., "Density Measurements by Means of Schlieren Methods,” April, 2003.

${ }^{15}$ Liepman, H. W., and Roshko, A., Elements of Gasdynamics Wiley, New York, 1985.

16 Viscous Interaction Performance Evaluation Routine for Two-Phase Nozzle Flows with Finite Rate Chemistry," Viper 3.6 User's Guide, Software and Engineering Associates, Inc., 2004. 
${ }^{17}$ Yim, J. T., et al., "Green Propellant Infusion Mission Plume Analysis Correlation with Plume Characterization Testing," JANNAF Conference Paper 2015-3883, June, 2015.

${ }^{18}$ Deans, M.C., et al., "Green Propellant Infusion Mission Thruster Perfoamcne Testing for Plume Diagnostics," 50th AIAA/ASME/SAE/ASEE Joint Propulsion Conference, Cleveland, OH, AIAA-20143483, July 2014.

19 "Ultra-High-Speed, Time-Resolved Spontaneous Raman Scattering Spectroscopy in Combustion," PI_emICCD_combustion_appnote_6_13_A1.pdf, Princeton Instruments, Inc. 2013.

${ }^{20}$ Clem, M. M., Brown, C. A., and Fagan, A. F., "Background Oriented Schlieren Implementation in a JetSurface Interaction Test," 51 ${ }^{\text {st }}$ AIAA Aerospace Sciences Meeting, AIAA Paper 2013-0038, January 2013. 
Table 1 Parameters of Raman Spectra for Molecules of Interest. ${ }^{6,8,9,11}$,Error! Bookmark not defined.

\begin{tabular}{|c|c|c|c|c|c|}
\hline \multirow{2}{*}{ Species } & \multirow{2}{*}{$\begin{array}{l}\text { Wavenumber } \\
\text { shift, } \mathrm{cm}^{-1}\end{array}$} & \multicolumn{2}{|c|}{$\begin{array}{l}\text { Wavelength shift, } \\
\text { for } \lambda_{\text {laser }}=532 \mathrm{~nm}\end{array}$} & \multicolumn{2}{|c|}{$\begin{array}{c}\text { Cross Sections } \\
\text { for } \lambda_{\text {laser }}=532 \mathrm{~nm} \\
10^{-30} \mathrm{~cm}^{2} / \mathrm{sr} \\
\end{array}$} \\
\hline & & Vibrational & $\begin{array}{c}\text { Rotational } \\
\text { (peak at } 700 \\
{ }^{0} \mathrm{C} \text { ) }\end{array}$ & Vibrational & Rotational \\
\hline $\mathrm{N}_{2}$ & 2331 & 607.3 & 535.6 & 0.46 & 3.65 \\
\hline $\mathrm{O}_{2}$ & 1556 & 580.0 & 534 & 0.47 & 12.13 \\
\hline $\mathrm{H}_{2}$ & 4160 & 681.2 & 565,580 & 0.943 & 0.71 \\
\hline \multirow{2}{*}{$\mathrm{C}_{2}$} & 2924 & 630.0 & 543 & 0.45 & 1.0 \\
\hline & 3172 & 640.0 & & 0.38 & - \\
\hline \multirow{2}{*}{$\mathrm{CO}_{2}$} & 1388 & 578.4 & 533 & 0.6 & 41.3 \\
\hline & 1285 & 571.0 & - & 0.45 & - \\
\hline \multirow{2}{*}{$\mathrm{NO}_{2}$} & 1320 & 572.2 & - & 7.37 & - \\
\hline & 754.0 & 554.2 & - & 3.63 & - \\
\hline $\mathrm{CO}$ & 2145 & 600.5 & 530 & 0.48 & 0.27 \\
\hline $\mathrm{H}_{2} \mathrm{O}$ & 3657 & 659.3 & - & 0.9 & - \\
\hline \multirow{2}{*}{$\mathrm{CH}_{4}$} & 2915 & 629.6 & - & 2.6 & - \\
\hline & 3017 & 633.7 & - & 1.7 & - \\
\hline NO & 1877 & 591.0 & - & 0.2 & - \\
\hline \multirow{2}{*}{$\mathrm{N}_{2} \mathrm{O}$} & 1282 & 570.9 & - & 0.98 & - \\
\hline & 2223 & 603.4 & - & 0.19 & - \\
\hline \multirow{2}{*}{$\mathrm{NH}_{3}$} & 3334 & 646.7 & - & 1.3 & - \\
\hline & 964.3 & 560.8 & - & & - \\
\hline $\mathrm{OH}$ & 3740 & 664.1 & & 0.9 & \\
\hline \multirow{2}{*}{$\mathrm{C}_{2} \mathrm{H}_{4}$} & 3020 & 633.8 & - & 1.9 & - \\
\hline & 1623 & 582.3 & - & 0.76 & - \\
\hline $\mathrm{C}_{2} \mathrm{H}_{6}$ & 993.0 & 561.7 & - & 0.69 & - \\
\hline \multirow{2}{*}{$\mathrm{C}_{6} \mathrm{H}_{6}$} & 3070 & 635.9 & - & 3.7 & - \\
\hline & 991.0 & 561.6 & - & 5.6 & - \\
\hline \multirow{4}{*}{$\mathrm{N}_{2} \mathrm{O}_{4}$} & 1400 & 574.8 & - & 0.28 & - \\
\hline & 800.0 & 555.7 & - & 0.15 & - \\
\hline & 1714 & 585.4 & - & 0.15 & - \\
\hline & 482.0 & 546.0 & - & 0.05 & - \\
\hline \multirow{3}{*}{$\mathrm{HNO}_{3}$} & 1050 & 563.5 & - & 7.4 & - \\
\hline & 1300 & 571.5 & - & 3.0 & - \\
\hline & 650.0 & 551.1 & - & 1.1 & - \\
\hline
\end{tabular}




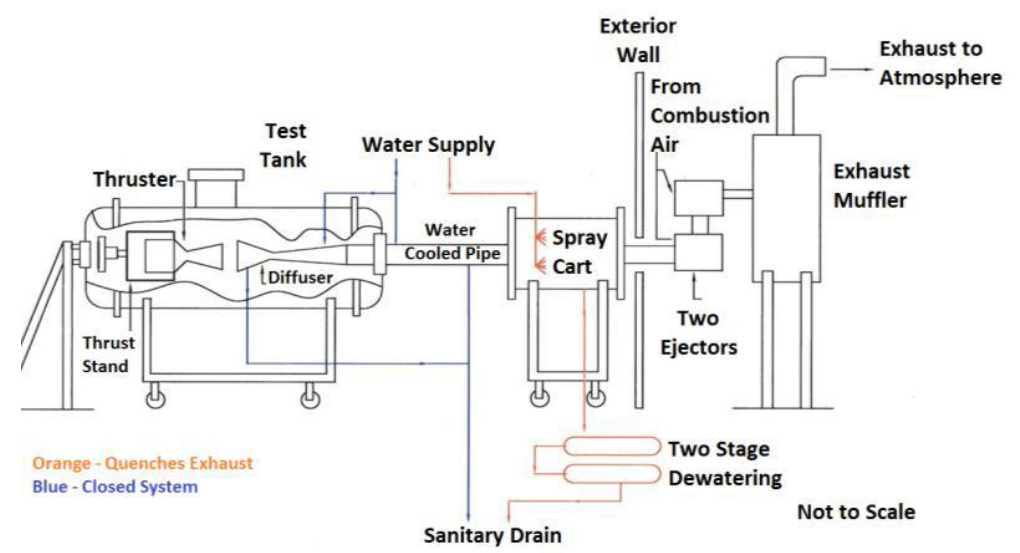

Figure 1 Schematic of the RCL-11 test chamber.

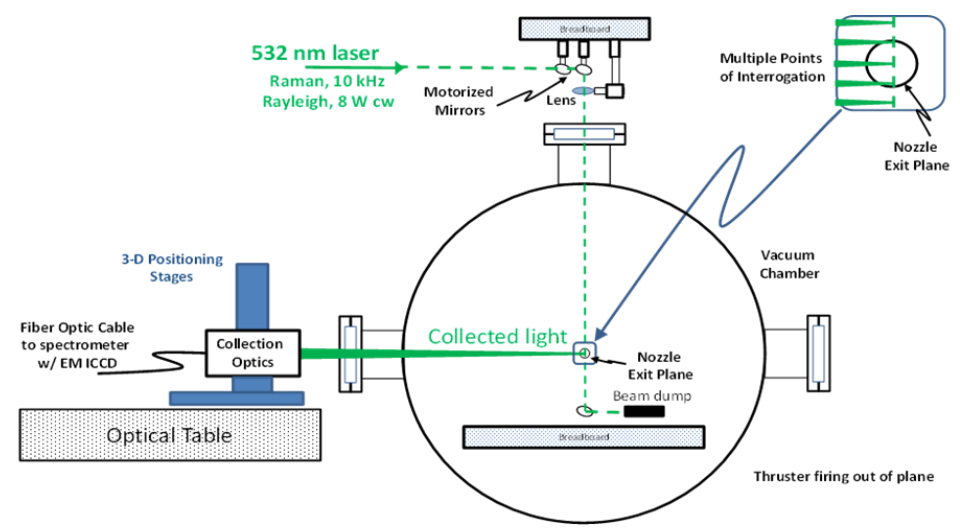

Figure 2 Schematic of laser delivery to the test facility.

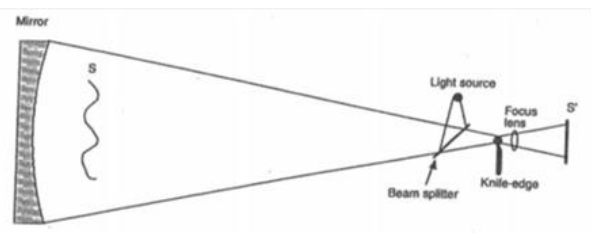

Single mirror coincident system

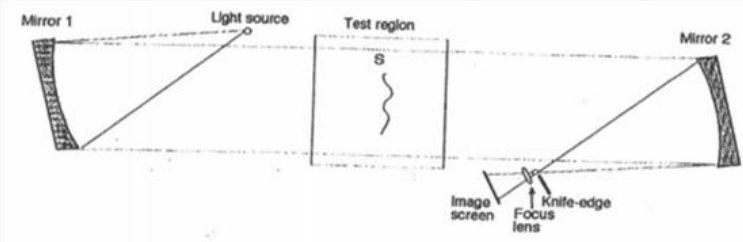

Z-type 2-mirror system

Figure 3 Schematic of Schlieren optical configurations. ${ }^{13}$ The single-mirror coincident configuration was used in this investigation. 


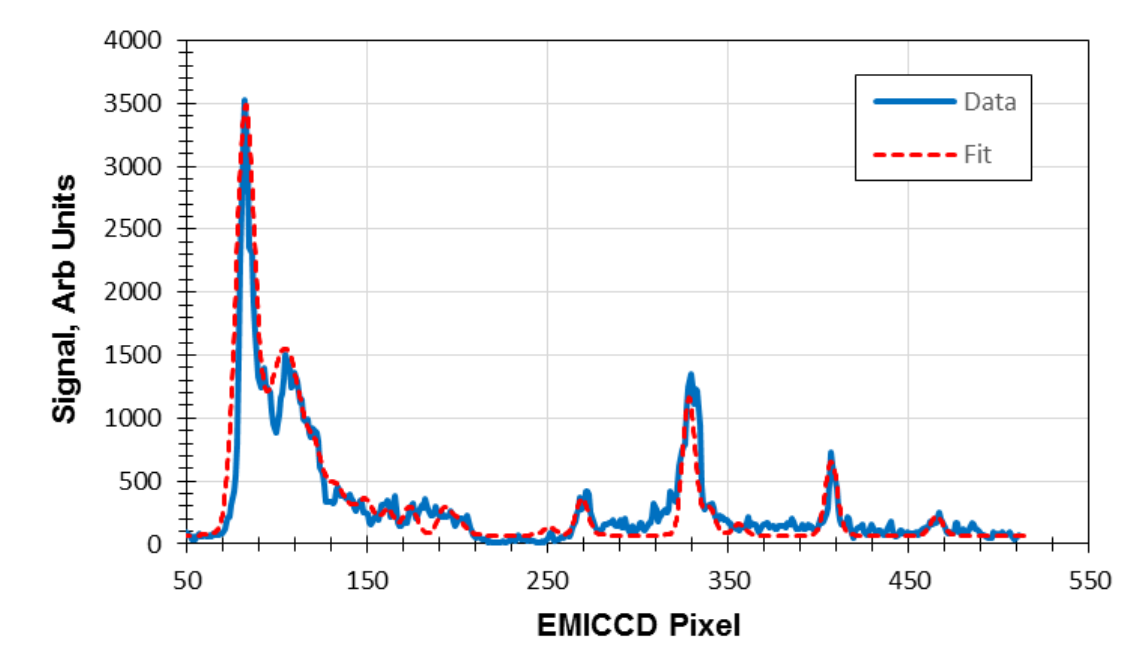

Figure 4 Typical mid-life Raman data and fit.

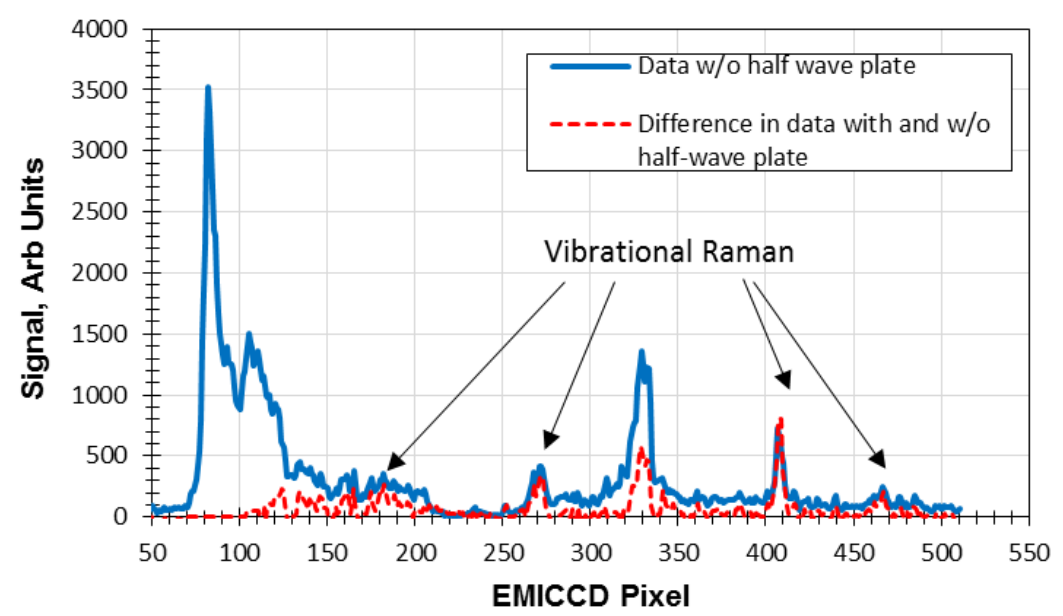

Figure 5 Vibrational-Raman signals remaining when the non-polarized signal (rotational-Raman and LIF) is subtracted from the total collected signal (without half-wave plate).

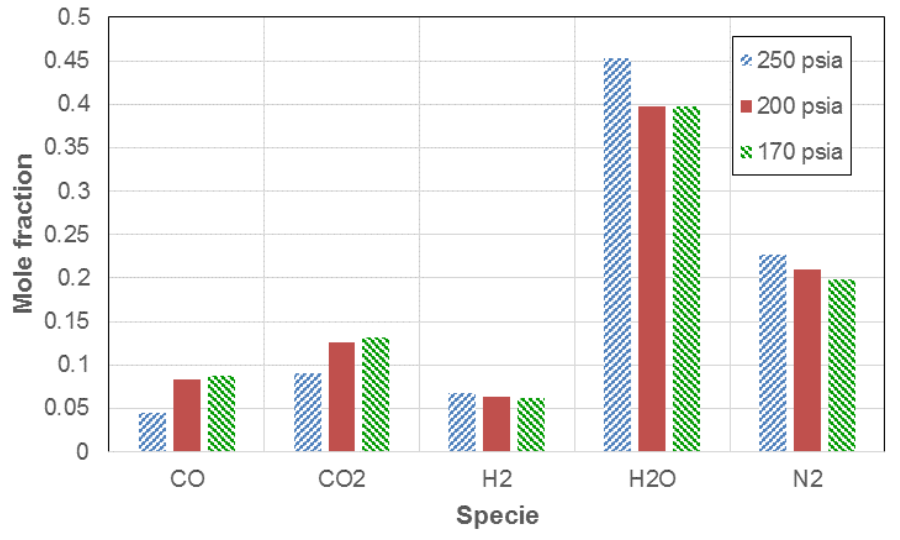

Figure 6 Trends as a function of chamber pressure, Pc, at catalyst mid-life. 


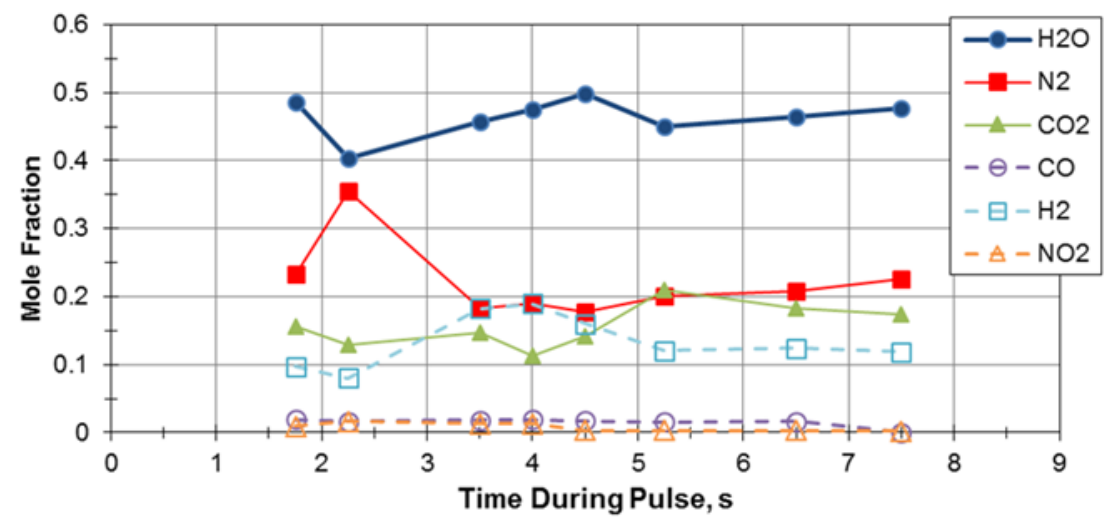

Figure 7 Variation of major species during an $8 \mathrm{~s}$ pulse at catalyst mid-life.
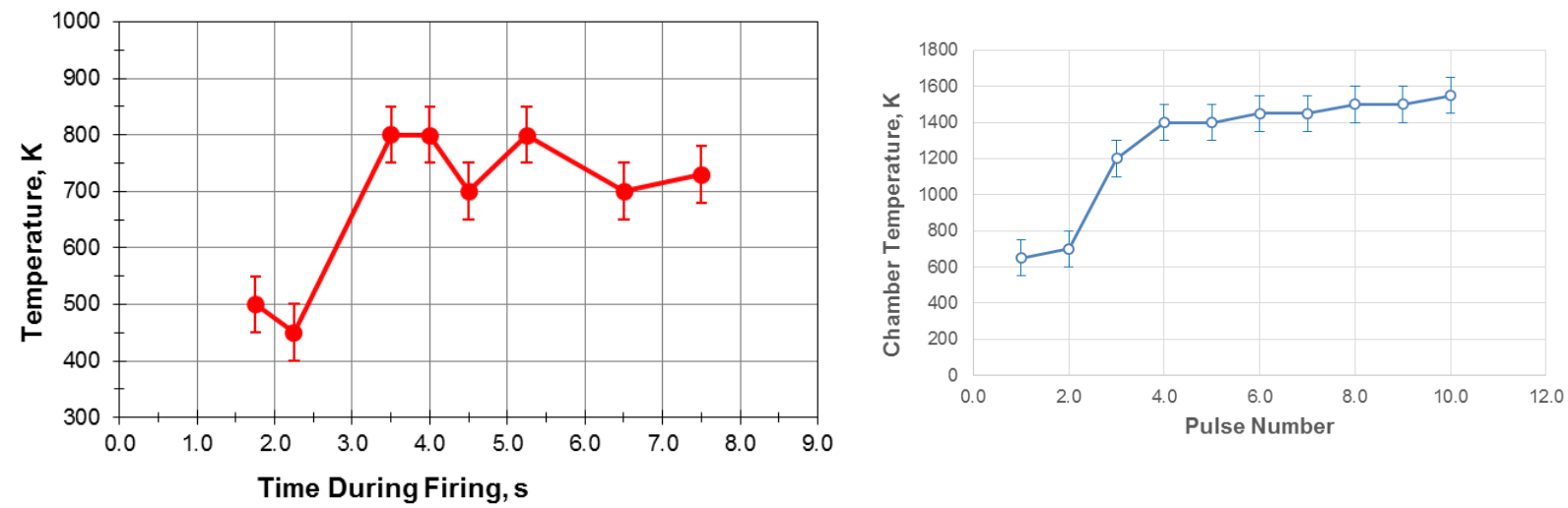

a. Raman-measured at $3 \mathrm{~mm}$ downstream of nozzle exit

b. Chamber temperature, Tc.

Figure 8 Trends in plume temperature and chamber temperature during an $8 \mathrm{~s}$ pulse at catalyst mid-life. 


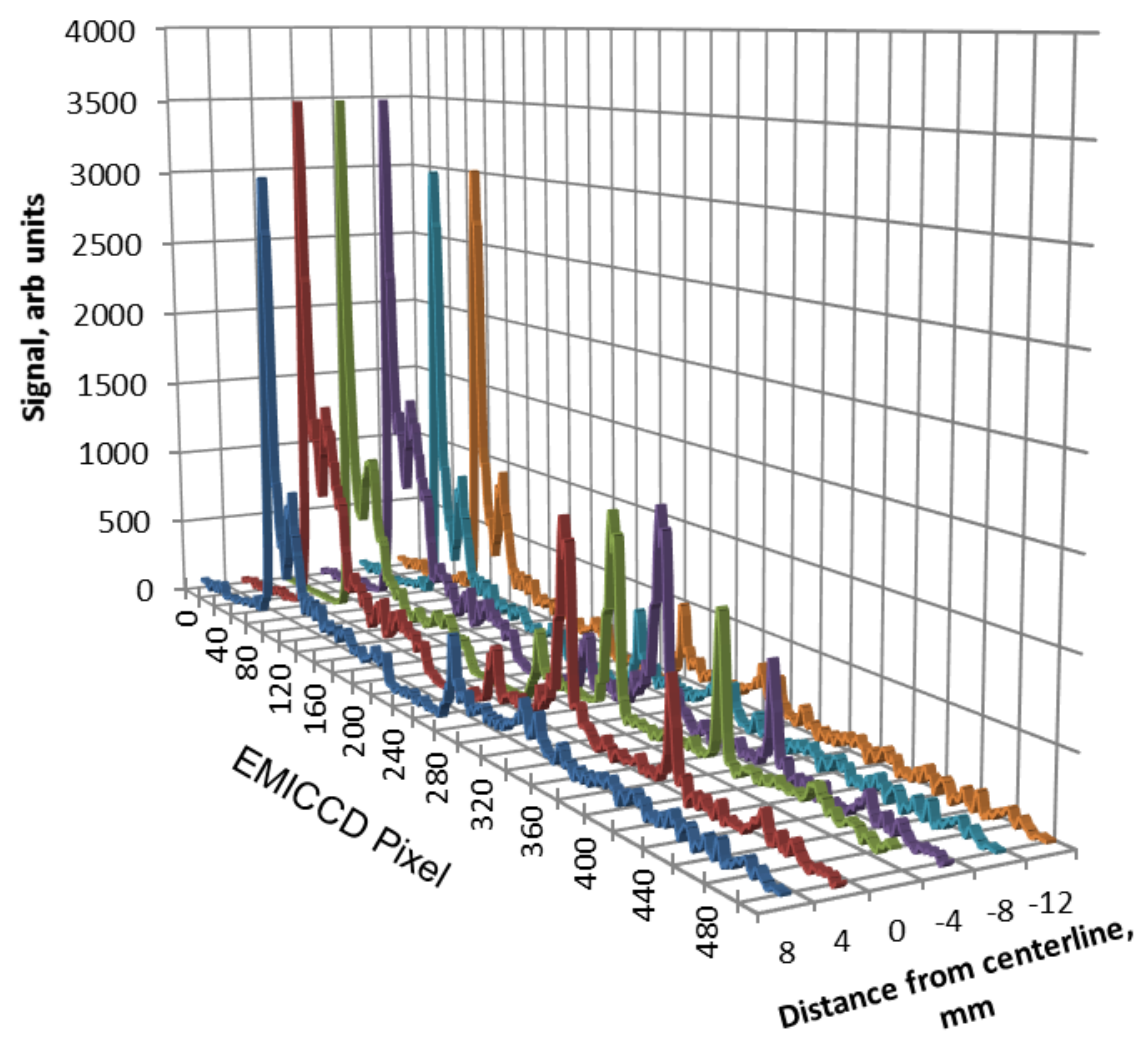

Figure 9 Spectra collected simultaneously at 6 different radial locations $5 \mathrm{~mm}$ downstream of the nozzle exit plane.

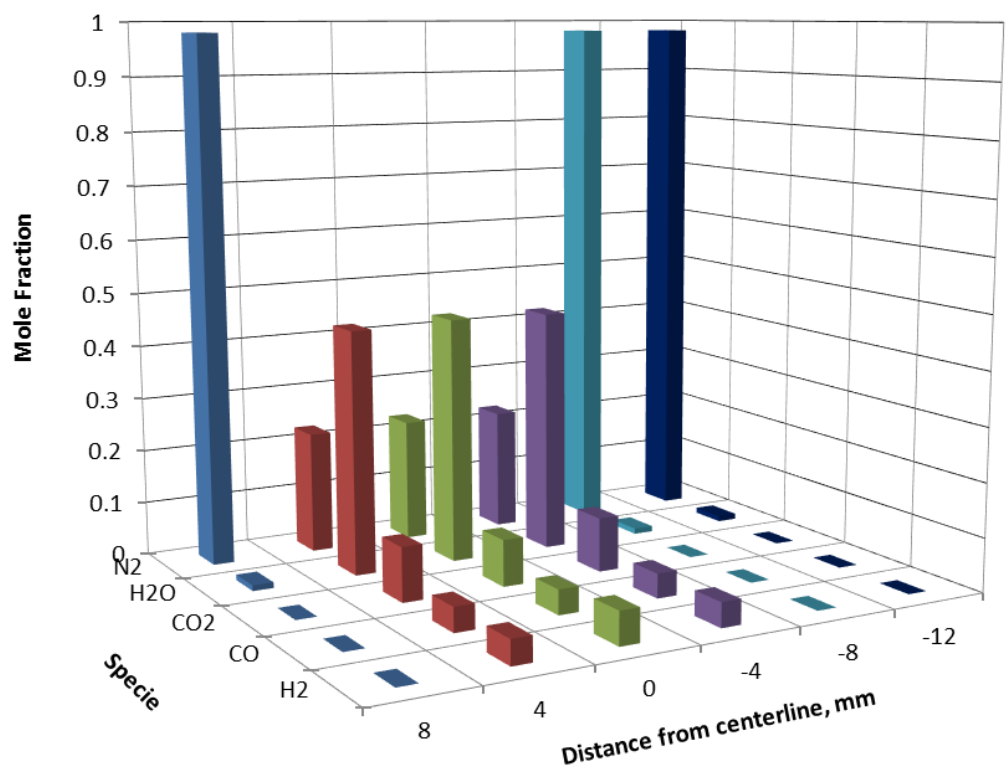

Figure 10 Concentrations at 6 radial locations based on the spectra in Fig. 9. 


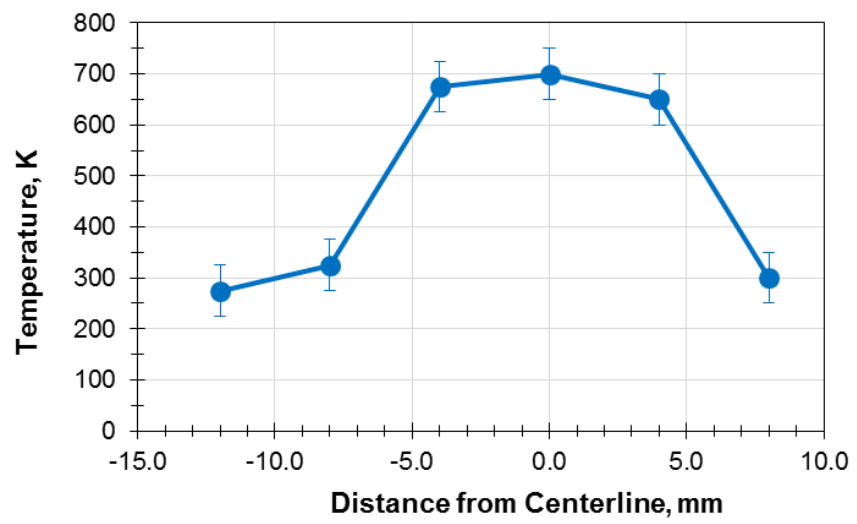

Figure 11 Radial variation in temperature measured $5 \mathrm{~mm}$ downstream of the exit plane.

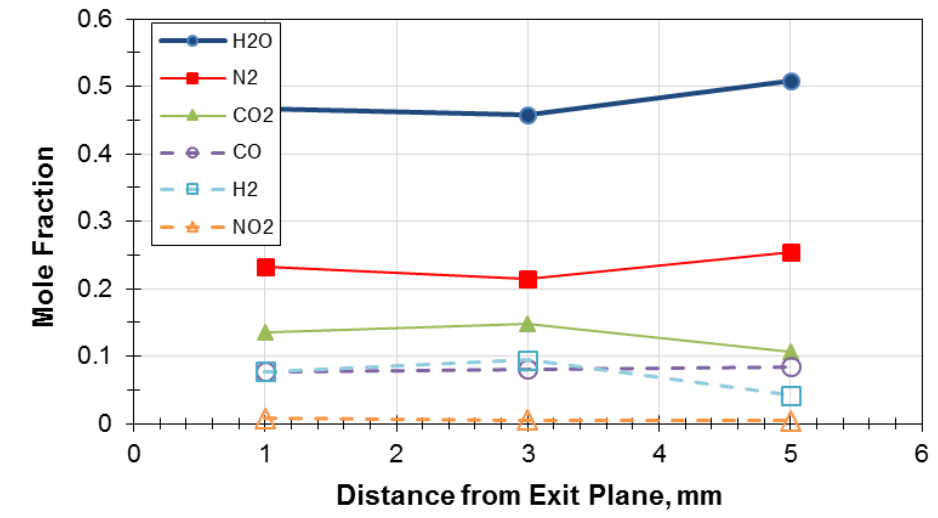

Figure 12 Variation in concentrations as a function of distance downstream of the nozzle exit plane.

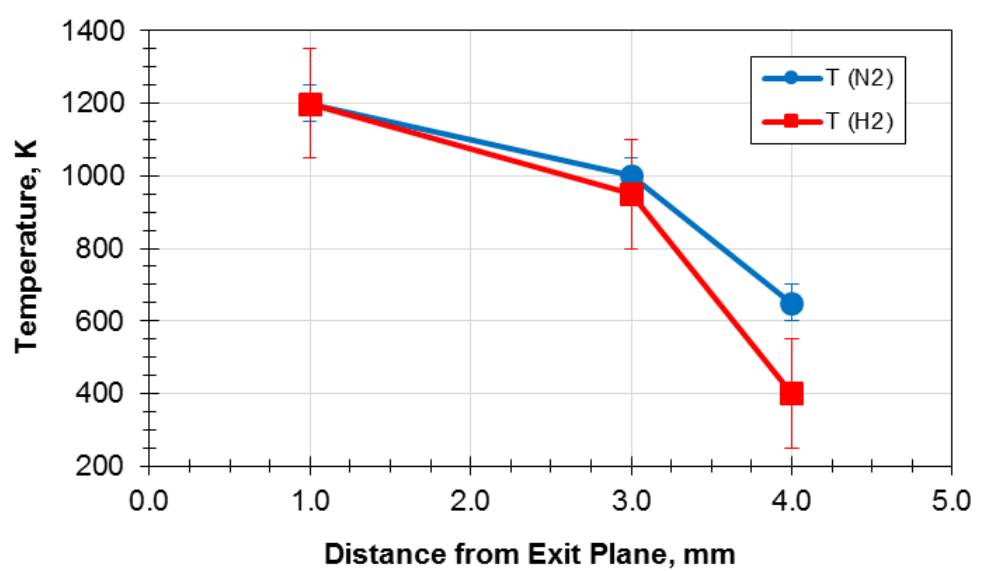

Figure 13 Variation in temperature as a function of distance downstream of the nozzle exit plane. 


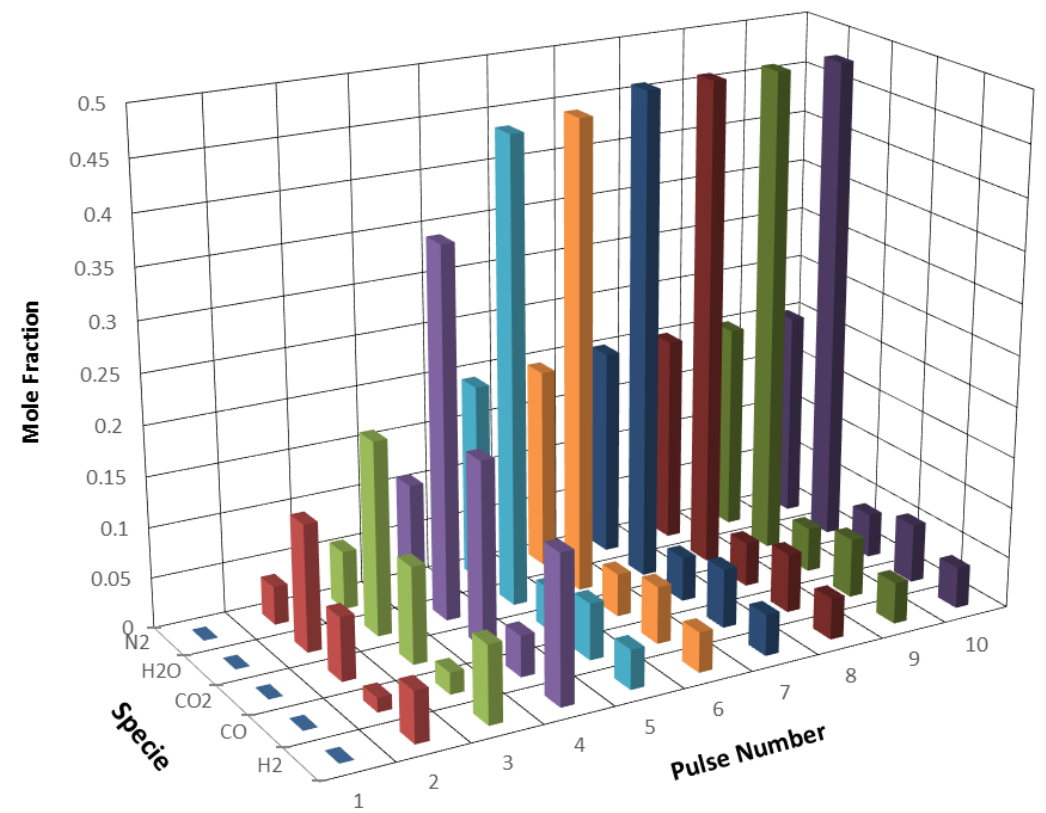

Figure 14 Variation in concentrations during a pulse train at catalyst mid-life.

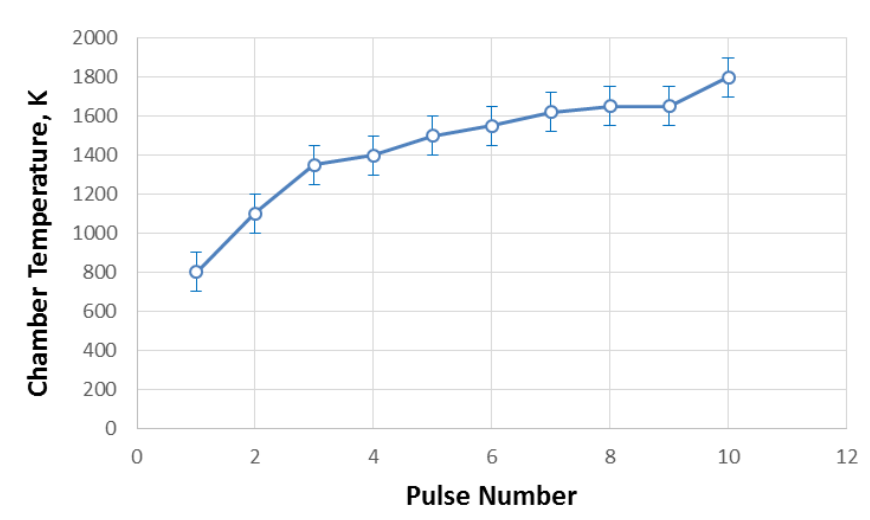

Figure 15 Variation in chamber temperature during a pulse train at catalyst mid life. 


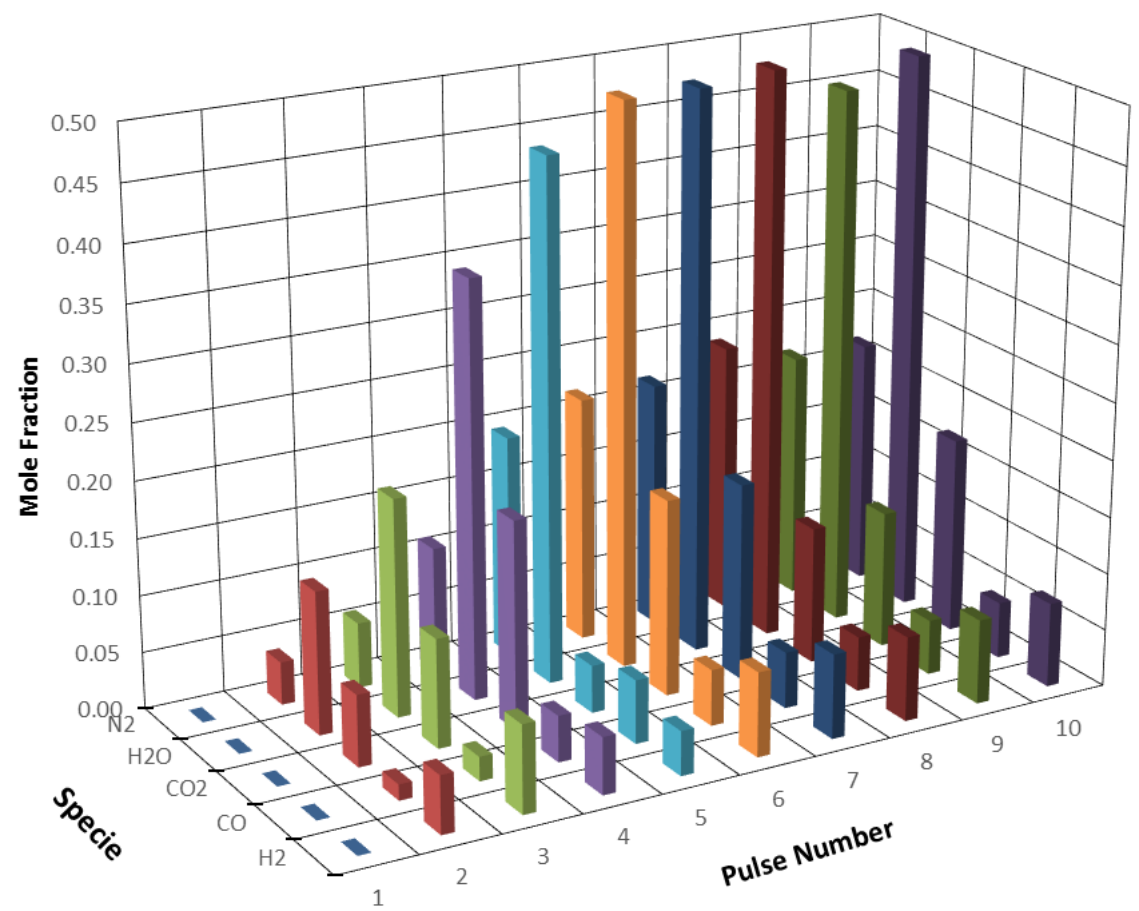

Figure 16 Variation in concentrations during a pulse train near catalyst end-of-life.

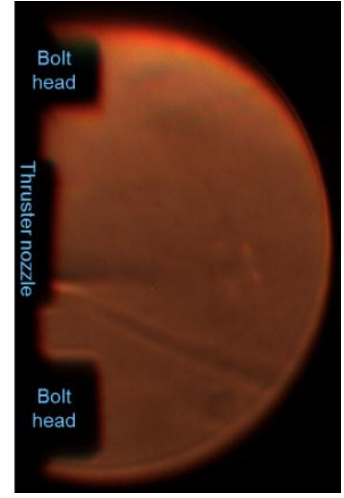

a. Raw schlieren image

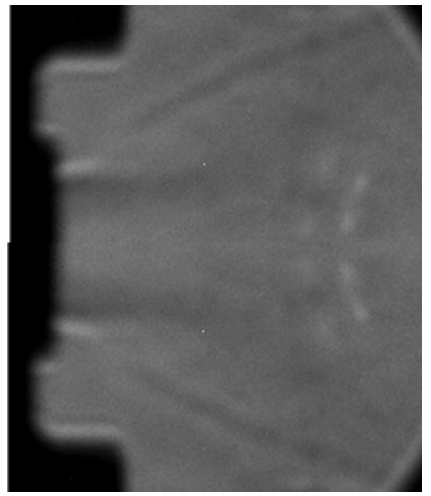

b. Enhanced schlieren image.

Figure 17 Typical Schlieren raw and enhanced images. 


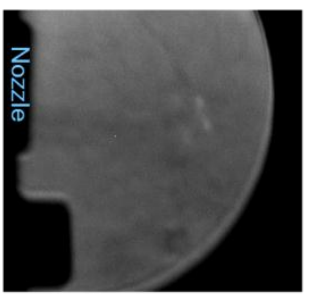

$t=-0.05 \mathrm{~s}$

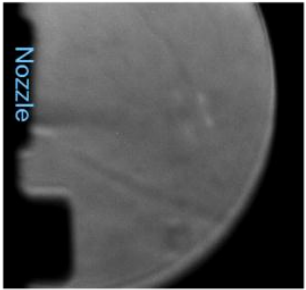

$\mathrm{t}=3.7 \mathrm{~s}$

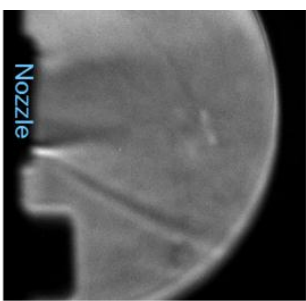

$t=0.15 \mathrm{~s}$

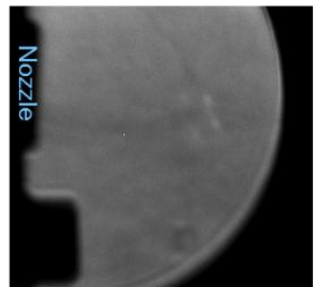

$\mathrm{t}=4.15 \mathrm{~s}$

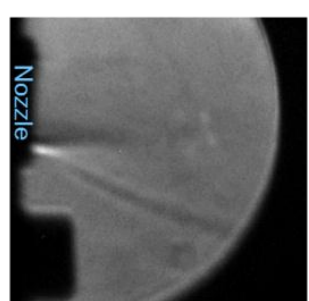

$t=1.3 \mathrm{~s}$

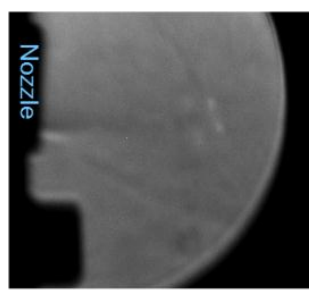

$\mathrm{t}=1.8$

Figure 18 Series of schlieren images showing variation in plume structure over a 4-s firing near catalyst end of life. $\mathrm{Pc}=200$ psia.

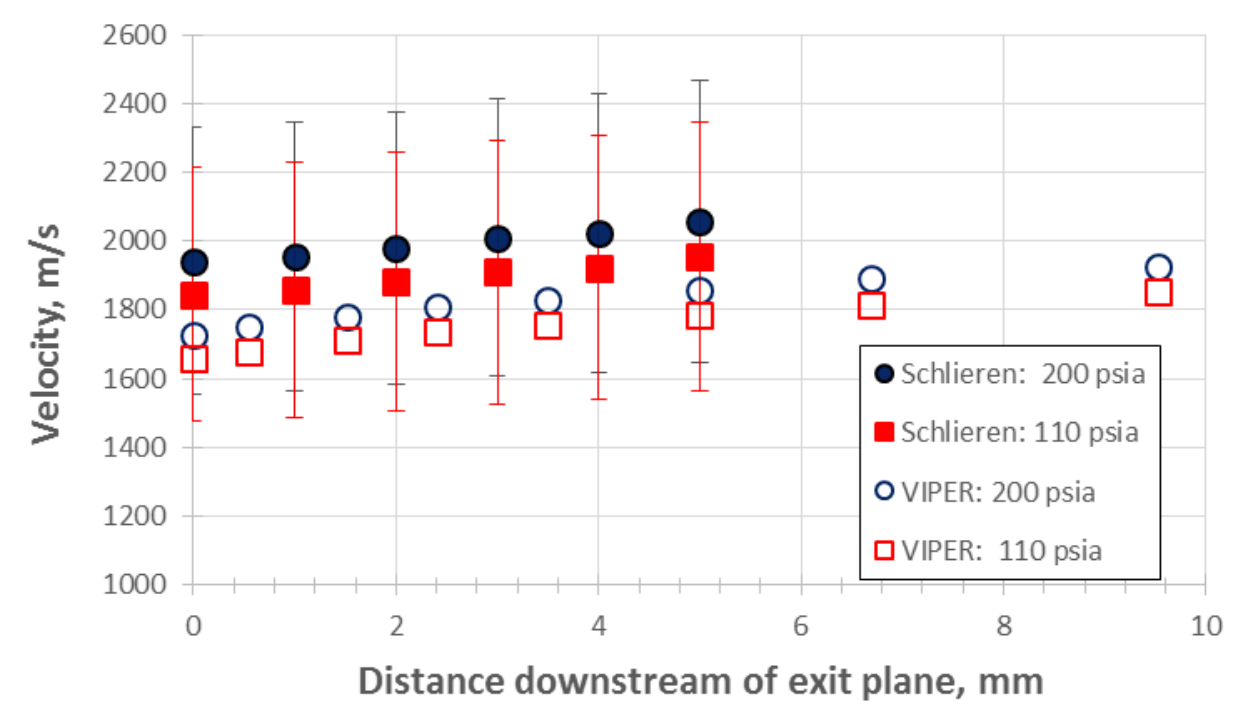

Figure 19 Velocity as a function of distance from nozzle exit plane calculated from schlieren images for chamber pressures of 110 and 200 psia. Calculated values using the VIPER code are also shown. 